
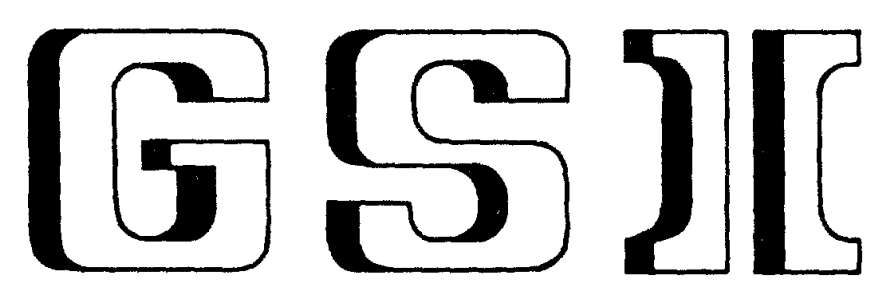

GSI-Preprint-96-22

MAI 1996

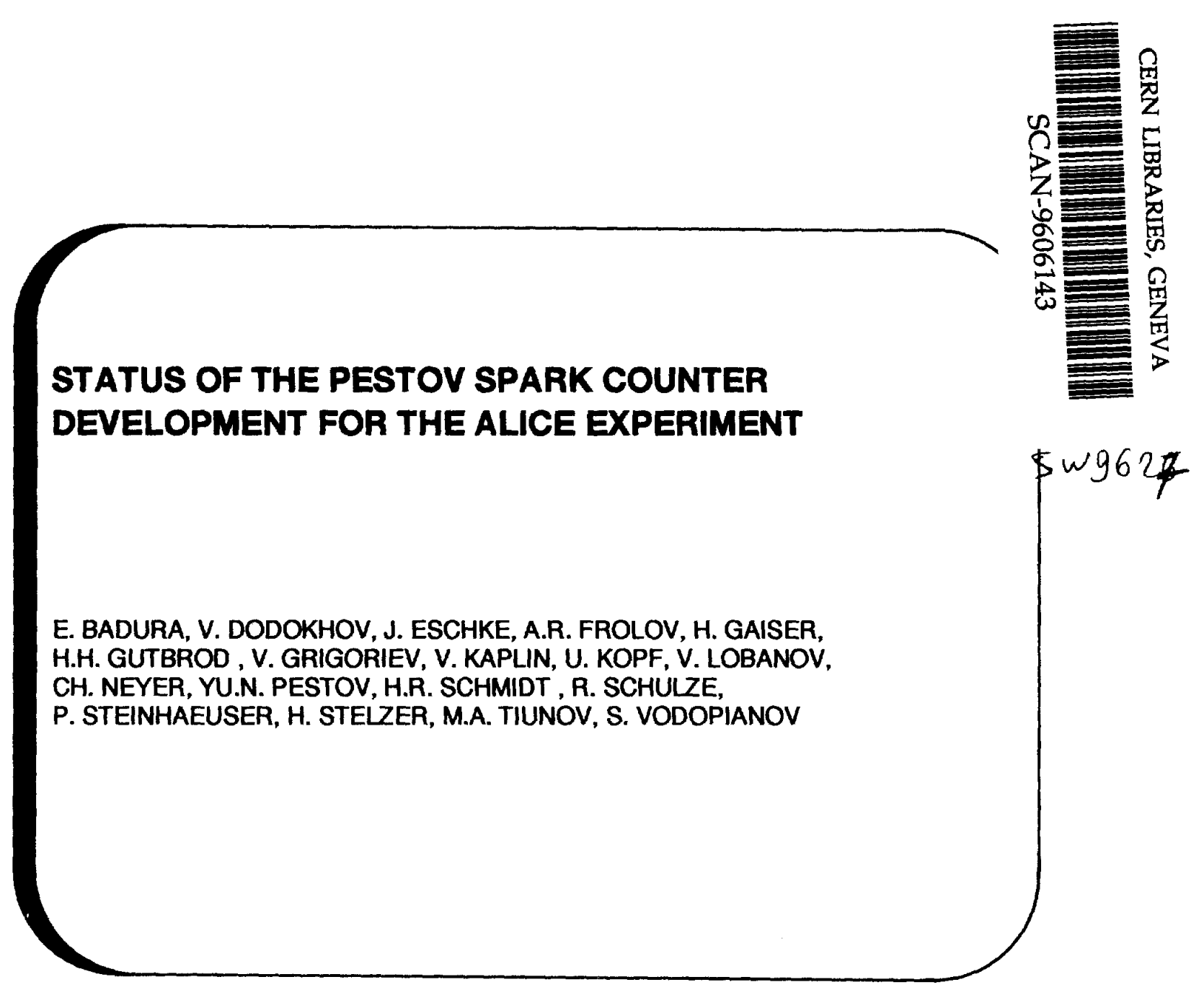

Gesellschaft für Schwerionenforschung $\mathrm{mbH}$ Postfach 110552 - D-64220 Darmstadt · Germany 


\title{
Status of the Pestov Spark Counter Development for the ALICE Experiment
}

\author{
E. Badura ${ }^{a}$, V. Dodokhor ${ }^{c}$, J. Eschke $^{a}$, A.R. Frolov ${ }^{b}$, H. Gaiser ${ }^{a}$, \\ H.H. Gutbrod ${ }^{a} 1$, V. Grigorier ${ }^{d}$, V. Kaplin ${ }^{d}$, U. Kop ${ }^{a}$, V. Lobanov ${ }^{c}$, \\ Ch. Neyer ${ }^{a}$, Yu.N. Pestor ${ }^{b}$, H.R. Schmidt ${ }^{a}{ }^{2}$, R. Schulze ${ }^{a}$, P. Steinhaeuser ${ }^{a}{ }^{3}$, \\ H.Stelzer ${ }^{a}$, M.A. Tiunor ${ }^{b}$, S. Vodopianor ${ }^{c}$ \\ a. Gesellschaft für Schwerionenforschung, Darmstadt, Germany \\ b. Budker Institute of Nuclear Physic, Novosibirsk, Russia \\ c. Joint Insitute for Nuclear Research, Dubna, Russia \\ d. Moscow Institute of Engineering Physics, Moscow, Russia
}

\begin{abstract}
The performance of a new ALICE prototype Pestov Spark Counter was measured both in dedicated test beam as well as in a high multiplicity heavy-ion environement. Results in terms of time resolution and background behaviour are presented.
\end{abstract}

\section{Introduction}

The Pestov Spark Counter [1,2], schematically shown in Fig. 1, is a single-gap, gaseous parallel-plate detector working in the streamer/spark mode. It makes use of a semi-conductive anode and of a special gas mixture that keeps the discharge local, thus increasing the rate capability. The spark discharges only a limited area of the semi-conductive anode $\left(\sim 12 \mathrm{~mm}^{2}\right)$, while the remaining area of the anode remains sensitive. 1 time resolution as good as $25 \mathrm{ps}$ (r.m.s.) is possible at wice the threshold voltage $(\sim 6 \mathrm{kl})$. The counter operates at a pressure of 12 bar, which vields 15 primary electrons from a minimum ionizing particle (MIP). The counter is read out via strip lines, which allows a two-dimensional reconstruction of the spark location.

\section{Design and Mechanical Construction of the Counter}

The basic Peston detector module is shown in lig. 2. It consists of two electrodes of $300 \mathrm{~mm}$ length, $10.68 \mathrm{~mm}$ width and $2 \mathrm{~mm}$ thickness. The gap

\footnotetext{
1 permanent address: SLBATECII, Nantes, France

2 corresponding author: Tel + 196159712655, F.LX +196159712989

3 permanent address: Iniversity of Karlruhe, Karlruhe, Germany
} 


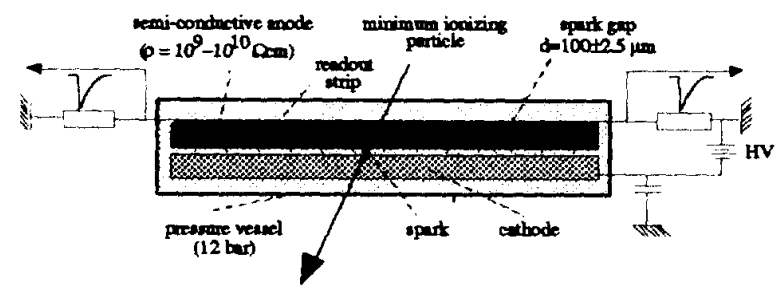

Fig. 1. Schematic layout of the Pestov Spark detector

between the electrodes is kept at $100 \mu \mathrm{m}$ by small spacers. The two electrodes are embedded in a plastic support structure with integrated gas channels which maintain a constant gas flow through the gap. The cathode is placed on the upper, thinner part of the support $(0.4 \mathrm{~mm}$ plastic $)$, which acts as a spring and ensures gas tightness by pressing the anode and cathode against the readout board. On top of the semi-conductive anode is a readout board with strip lines, which picks up the signal and transports it to the front-end electronics. It is glued into the flange and acts as a gas seal between the gas channels and forces the gas flow through the gap. At the same time it gives the system, together with the plastic support, mechanical stability. The pressure vessel, which houses the counter, is made out of an aluminum tube of $50 \mathrm{~mm}$ diameter and has a wall thickness of $1 \mathrm{~mm}$. $A$ cap is welded onto the tube on one side; the other side is connected to a flange. Several of these tubes have been tested at pressures of up to $100 \mathrm{bar}$ without rupturing. The anode is made from semi-conductive glass (resistivity $\sim 10^{9}-10^{10} \Omega \mathrm{cm}$ ) which is optically polished on both sides. The edges are specially shaped $\left(y=x^{4}\right)$ to reduce the electric field in this region. Several technologies and materials have been tested for producing a high-quality cathode surface. The most promising seems to be sputtering of a copper film on standard float glass, which has been used for detectors presented here. $A$ grid $(d=22 \mathrm{~mm})$ of spacers is manufactured into the semi-conductive anode in order to keep the sagitta due to the electrostatic pressure below $\sim 0.2 \%$ of the gap distance. These spacers, glass balls of $1 \mathrm{~mm}$ diameter, are glued into depressions of $0.9 \mathrm{~mm}$ depth and are adjusted by polishing to a gap width of $100 \mu \mathrm{m}$. The edges of the depressions are also $x^{4}$-shaped to avoid electrical breakdown [3].

\section{Production of Semi-Conductive Pestov Glass}

The possibility to produce semi-conductive glass with a bulk resistivity in the range of $10^{9}$ to $10^{10} \Omega \mathrm{cm}$ by industrial methods is a key issue for any large scale application of the Pestor spark counter. R\&D efforts to find means of mass production of semi-conductive glass where undertaken both with SCIIOTT, Mainz and LF.WZOS, St. Petersburg. The glass produced at the two companys differs in its composition, i.e. at LENZOS a mixture with lower $Z$ components was investigated. The result in terms of bulk resistivity of the test meltings 


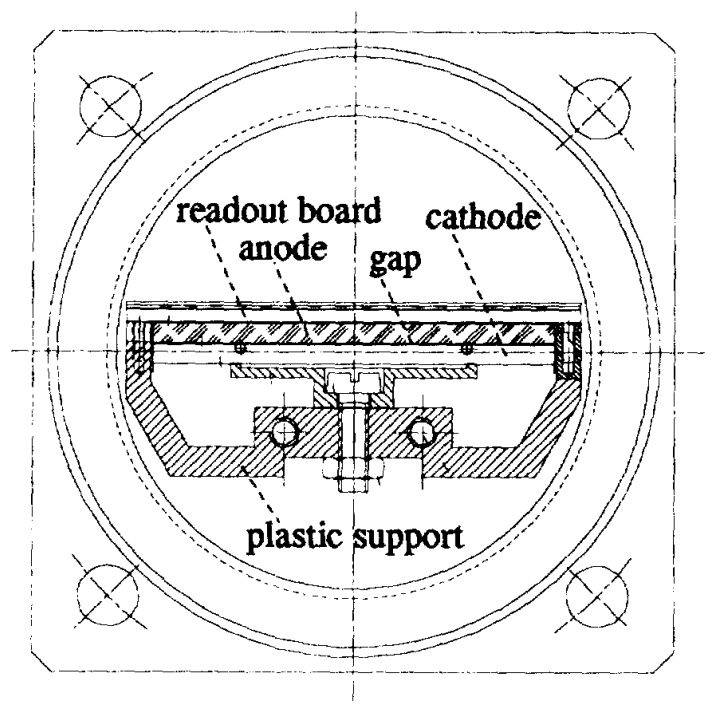

Fig. 2. Cross-section of a single Pestov Counter

carried out at the two companies is shown in Fig. 3, together with resistivity measurements at samples from laboratory production of Pestov glass at Moscow. As can be seen the respective resistivities fall all in the right "window" in which the glass can be used for application in high energy experiments. Moreover, the four samples from SCHO'T result from consecutive meltings carried out under the same, controlled conditions, showing that the technology employed yields reproducible results.

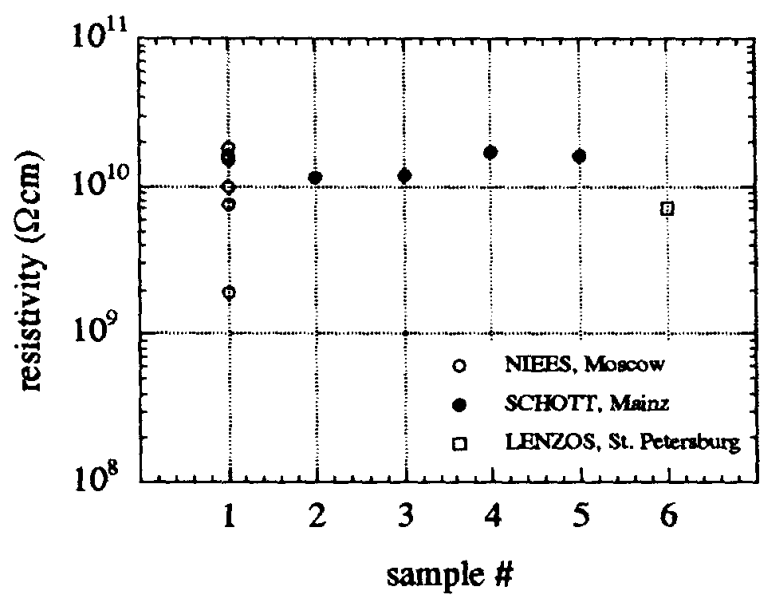

Fïg. 3. Bulk resistivity for samples from different meltings of semi-conductive Pestov glass 


\section{Double Threshold Discriminator}

For the Pestov counter a fast leading-edge discriminator is essential, since the excellent timing information is contained in the first $300 \mathrm{ps}$ of the pulse. Moreover, due to after-pulses, the trailing part of the signal varies and may even have secondary maxima. Standard leading-edge discriminators limit the time resolution to $\sim 70 \mathrm{ps}$, as no amplitude-dependent walk correction is possible with the irregular pulse shapes. In the PS test a novel type of double threshold of disriminator (DTD) was used for the first time. The basic idea, i.e. the discrimination of the input pulse at two thresholds with hardware extrapolation to $t_{0}$, as well as the principal circuit diagramms are developed in Ref. [7]. The practical realization for the present detector is described in Ref. [8].

\section{Test Results}

The data presented in the paper show mainly results obtained in recent measurements both at the CERN PS test beam as well as from the CERN SPS, where the counter was installed within the NAt9 experiment. The condition in the latter test are, as far as counting rate and occupancy are concerned, very similar to the expected ALICE running conditions. With the data analysis still ongoing, the (preliminary) results shown below are concerned with efficiencies, background behaviour and time resolution. Results on longterm stability were published recently elsewhere [ $t]$. On the present level of analy'sis no degradation of the counter performance was experienced when operated in the high multiplicity environnement in the NA 49 experiment.

\subsection{Efficieny, Plateau Curve and Background}

The efliciency is both given by the intrinsic properties of the counter, i.e. by the number of primary ionizations and the charge released by the spark, as well as by the quality of the signal transmission to the discriminators. In particular the new double threshold discriminator requires, due to the second, relatively high threshold $\left(10 \mathrm{ml}, 20 \mathrm{~m} \mathrm{I}^{\circ}\right.$ for the lower threshold), minimal damping of the signal induced by the spark. The efficiency, obtained from correlating two counters is shown in Fig. 1. The figure displays the results from the PS run where the new readout geometry and the double threshold discriminator was employed for the first time, and efliciencies obtained in previous measurements at BNIP Vovosibirsk. Is can be seen, the values from the new counter coincide with the values from previous measurements. 


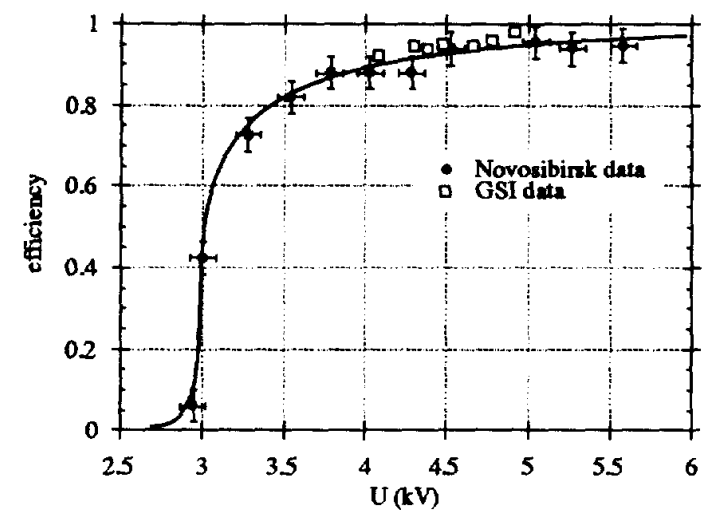

Fig. 4. Efficiency of the Pestov Spark counter measured at BNIP Novosibirsk [1] (filled circles) and in the present experiment(open squares)

The plateau curve for gamma rays is showm in Fig. 5 on the left side axis. The measurement was done after approximately 10 days of operation at the SPS in the NAt9 experiment. The curve exhibits a threshold value of $3.2 \mathrm{~kJ}$ and a flat plateau which extends from about $3.8 \mathrm{kV}$ up to $5.0 \mathrm{kV}$, indicating that the counter is, relative the rate induced by the gamma source, essentially background free. Closer inspection of the background rate by covering the gamma. source vields the very low rate of $0.2-0.3 \mathrm{~Hz} / \mathrm{cm}^{2}$ (right axis). However, the linear rise of the rate with voltage indicates that the background is generated intrinsically. Further improvements in the cathod production or/and cleaning technology might reduce the background to comic ray level $\left(<0.03 \mathrm{~Hz} / \mathrm{cm}^{2}\right)$; a background rate which has already been reached previously [H] for selected counter areas.

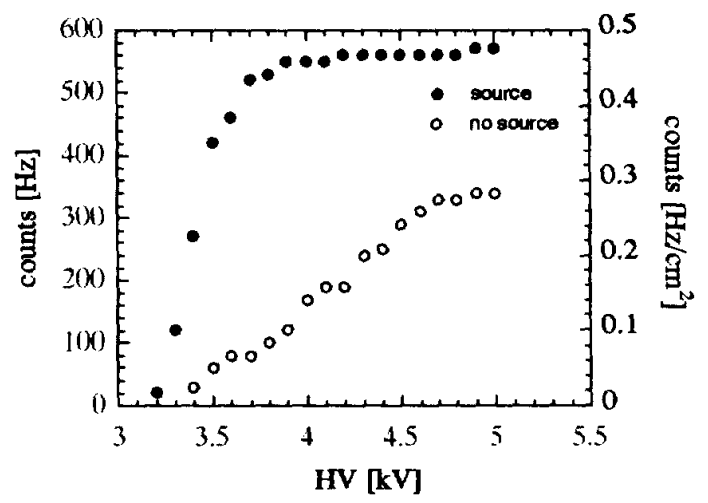

Fig. 5. Counting rate vs HV ("plateau curve") with (left side axis) and without (right side axis) a ${ }^{61} \mathrm{Co}$ gamma source irradiating the counter 


\subsection{Time Resolution}

The time resolution shown in Fig. 6 is obtained at the SPS run from correlating two Pestor counter, which were positioned behind each other. This resolution of sigma $<35 \mathrm{ps}$ is obtained locally, i.e.with cuts on the longitudinal extend of the irratiated area on the counter. It is, however, approximately independent on the position of this area on the counter. This feature seems also to be the reason for the non-gaussian tails of the time resolution as indicated by the comparision of the $\mathrm{dt}$ distribution with a gaussian curve fitted to the upper part of the spectrum. Preliminary investigations of the source this problem points toward cross talk in the discriminator board (two discriminators were mounted onto one printed circuit board and had been connected to the two ends of one readout stripline) giving rise to an (unphysical) time shift of up to $\pm 10 \mathrm{ps}[5]$. The shift is dependent on the position of the spark relative to the readout stripline thus spoiling the global time resolution. It should be remarked that recent measurements with another DTD prototype vielded, due to a different arrangement of the components on the printed circuit board, a significantly reduced cross talk [6].

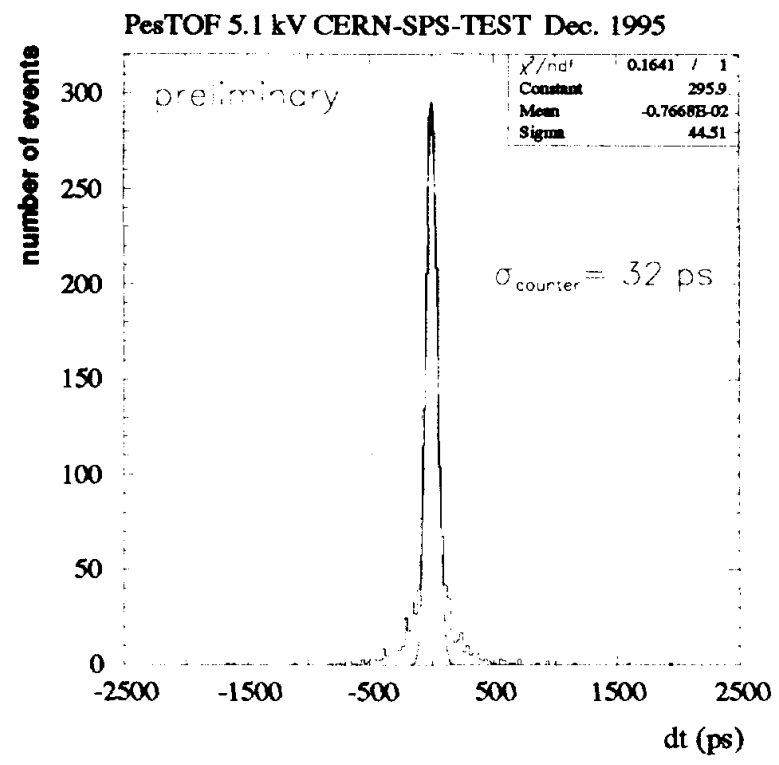

Fig. 6. Time resolution $\mathrm{dt}$ obtained at a voltage of $5.1 \mathrm{kV}$

A compilation of the time resolution obtained in the manner described above is shown in Fig. 7 and compared with results from previous measurement at B.NIP. As can be seen the resolution of the new counters, equipped with the DTD, drops faster with the HS applied and reached the level of $35-10 \mathrm{ps}$ at a IIV of about $0.7 \mathrm{k}$ l lower. This feature, due to the very good resolution of the I) TD. allows to reach the nominal time resolution for the NLCF experiment $(\approx 50 \mathrm{ps})$ at a much lower voltage then previously anticpated thus improving the lifetime of the counter considerably. Horeover, due to the reduced clectric 
field in the counter the susceptibility to mechanical imperfections is greatly reduced.

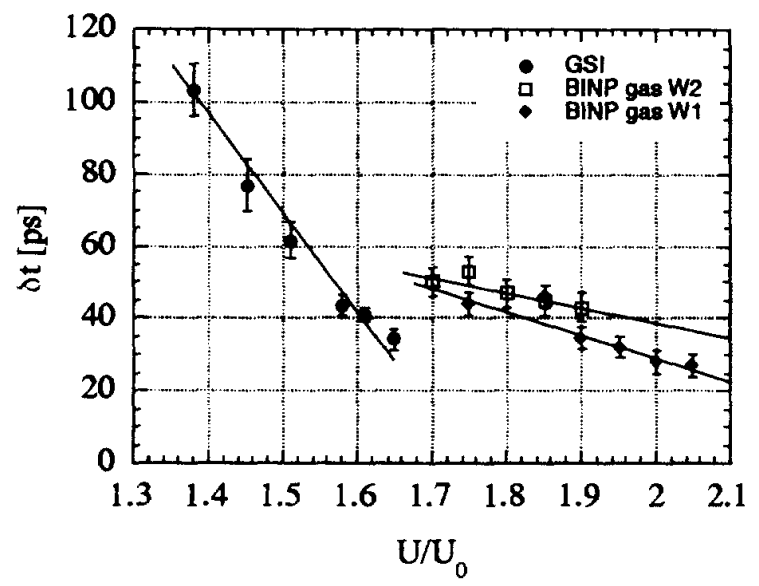

Fig. 7. Time resolution from the new prototype (filled circes) and from earlier mesurements done at BNIP Novosibirsk [1] (squares, diamonds)

\section{Conclusion}

In summary, we have build and tested a new prototype Pestor counter which has already most of the mechanical features forseen for its application in the ALICE experiment. Furthermore, both SCHO'TT, Mainz and LENZOS, St. Petersburg have been able to produce semi-conductive glass by industrial means. The efficiency $(97 \%)$, time $(<10 \mathrm{ps})$ and position $(<350 \mu \mathrm{m})$ resolution reach or are well beyond the requirements for $\triangle \mathrm{LICE}$. The background rate was found to be on the very low level of $0.2-0.3 / 1 \mathrm{z} / \mathrm{cm}^{2}$.

\section{References}

[1] Yu. P. Pestov, Proce th San Miniato Topical Seminar, World Scientific, 1991, p. 156 , and Ref, therein

[2] A.R. Frolor, Yu.N. Pestov and V.V. Primachek, Nucl.Inst. Methods A307 (1991) 497

[3] Yu. Pestov, M..1. Tiunov, Preprint BNIP 9477 (1991)

[4] E. Badura et al., Nucl. Inst. Methods 372 (1996) 352

[5] Ch. Never, private communication

[6] A. Frolov and Yu. Pestov, private communication

[7] A. Frolov, T.Y. Oslopova and Yu. Pestow, Inst. Methods 356 (1995) 497

[8] Ch. Veyer et al., ".I precise discriminator for Time-of-Flight measurements in ILICE:", Proceedings of the first workshop on Electronics for LHC Experiments, I.isbon (1995) 
